\title{
The Scattering Theory for the Nonlinear Wave Equation with Small Data, II
}

\author{
By \\ Kiyoshi MochizUKI* and Takahiro MOTAI**
}

\section{§0. Introduction}

In a previous paper with the same title [7], we studied a scattering problem for the nonlinear wave equation

$$
\partial_{t}{ }^{2} w(t)=\Delta w(t)+f(w(t))=0 \quad \text { in } \quad(x, t) \in \mathbb{R}^{n} \times \mathbb{R} .
$$

Here $n \geq 2$ and $\Delta=\sum_{j=1}^{n} \partial_{x_{j}}^{2}$. For purposes of illustration, let $f(w)=$ $\lambda|w|^{\rho-1} w(\lambda \in \boldsymbol{R}, \rho>1)$. It was then proved that the scattering operator exists on a dense set of a neighborhood of 0 in the energy space if $\rho$ is restricted to satisfy

$$
\begin{gathered}
\rho \geq 2 \text { and } \\
\gamma(n) \equiv \frac{n^{2}+3 n-2+\sqrt{\left(n^{2}+3 n-2\right)^{2}-8 n(n-1)}}{2 n(n-1)}<\rho \leq \frac{n+3}{n-1} .
\end{gathered}
$$

The lower bound $\gamma(n)$ of $\rho$ is smaller than those obtained in Strauss [10] and Klainerman [6], and it is expected to give a critical power. On the other hand, (0.2) is too restrictive. For it contradicts (0.3) in case of high space dimension $(n \geq 5)$. The main purpose of the present paper is to eliminate this restriction. Namely, in this paper we shall extend our previous results to all $\rho$ satisfying (0.3).

Communicated by S. Matsuura, October 13, 1986.

* Department of Mathematics, Faculty of Science, Shinsyu University, Matsumoto 390, Japan.

** Institute of Mathematics, University of Tsukuba, Sakura-Mura, Ibaraki 305, Japan.

Present address : Japanese Language School, The University of Tokyo of Foreign Studies, Fuchu 183, Japan. 
For the existence of the scattering operator, it is necessary to solve the Cauchy problem for $(0.1)$ with $-\infty$ initial time. Let $w_{0}^{-}(t)$ be a solution of the free wave equation

$$
\partial_{t}{ }^{2} w(t)-\Delta w(t)=0 \quad \text { in } \quad(x, t) \in \boldsymbol{R}^{n} \times \boldsymbol{R} .
$$

Then the integral version of this problem is given by

$$
w(t)=w_{0}{ }^{-}(t)-\int_{-\infty}^{t} H^{-1} \sin \{H(t-\tau)\} f(w(\tau)) d \tau,
$$

where $H=\sqrt{-\Delta}$ in $L^{2}\left(\boldsymbol{R}^{n}\right)$, or more generally, we define functions $\zeta(H)$ of $H$ as follows: Let denote the Fourier transformation and $\mathscr{I}^{-1}$ be its inverse. Then

$$
\zeta(H) u=\mathscr{T}^{-1}\left\{\zeta(|\xi| \hat{u}(\xi)\} \quad \text { in } \mathcal{S}^{\prime}\right. \text { (tempered distribution) . }
$$

Let \|\|$_{e}$ be the energy norm defined by

$$
\|u(t)\|_{e}=\frac{1}{\sqrt{2}}\left\{\|H u(t)\|_{2}{ }^{2}+\left\|\partial_{t} u(t)\right\|_{2}^{2}\right\}^{1 / 2},
$$

where $\|u\|_{p}(p \geq 1)$ is the usual $L^{p}$-norm. Let $H^{s, p}(s \in \boldsymbol{R}, p \geq 1)$ [resp. $\dot{H}^{s, p}$ $(s>-n, p>1)]$ be the Sobolev spaces which are the completion of $C_{0}{ }^{\infty}\left(\boldsymbol{R}^{n}\right)$ with norms

$$
\|u\|_{s, p}=\left\|\left(1+H^{2}\right)^{s / 2} u\right\|_{p} \quad\left[\text { resp. }\|u\|_{s, p}^{\cdot}=\left\|H^{s} u\right\|_{p}\right],
$$

and let

$$
V=V_{s, p}^{d} \equiv\left\{u(t) \in C_{t}\left(\boldsymbol{R} ; H^{s, q}\right) ;\|u\|_{V}=\sup _{t}(1+|t|)^{d}\|u(t)\|_{s, q}<\infty\right\},
$$

where

$$
q=\frac{2(n \rho-1)}{n+1}, \quad s=\frac{n+1}{q}-\frac{n-1}{2} \text { and } d=(n-1)\left(\frac{1}{2}-\frac{1}{q}\right) .
$$

We consider the integral equation (0.5) in this space $V$. Then a contraction mapping principle is applicable, and we have a unique solution $w(t) \in V$, which also satisfies

$$
\left\|w(t)-w_{0}^{-}(t)\right\|_{s, q} \rightarrow 0 \text { as } t \rightarrow-\infty .
$$

For the purpose of the scattering theory, however, $(0.10)$ is insufficient. What we like to show is

$$
\left\|w(t)-w_{0}^{-}(t)\right\|_{e} \rightarrow 0 \text { as } t \rightarrow-\infty .
$$

In [7] we treated (0.5) in the space $V_{1, q}^{d}$, where $q$ and $d$ are given above. 
In this case (0.11) directly follows from (0.5) since we have the embedding $H^{1, q} \hookrightarrow L^{2 \rho}$. However, to construct solutions in $V_{1, q}^{d}$, we require (0.2) which guarantees a Lipschitz continuity of $f^{\prime}(w)$.

In the present case, the embedding $H^{s, q} \hookrightarrow L^{2 \rho}$ is no longer expected to hold, instead we shall follow the method of Strauss [10], where (0.11) results from the energy inequality

$$
\|w(t)\|_{e}{ }^{2}+\frac{\lambda}{\rho+1}\|w(t)\|_{\rho^{\rho+1}}^{\rho+1} \leq\left\|w_{0}-(0)\right\|_{e^{2}} .
$$

Our main problem thus turns out to show this inequality. It does not come directly from the integral equation (0.5). So, we return to the differential equation (0.1) and apply an approximate energy method. The double convolution mollifier due to Ginibre-Velo [2] will give a convenient approximation of $f(w)$.

The paper is organized as follows : In $\S 1$ we summarize our results in Theorem. A proof of Theorem is given through the following three sections. In $\S 2$ is shown the existence of solutions. Energy estimates of solutions are obtained in $\S 3$. Finally, in $\S 4$ the proof is completed.

\section{$\S 1$. Assumptions and Theorem}

Throughout the paper, the nonlinearity $f(w)$ is real-valued and is required to satisfy

$$
\begin{aligned}
& f(w) \in C^{1}(\mathbb{R}) \text { and } f(0)=0 . \\
& \left|f^{\prime}(w)\right| \leq C|w|^{\rho-1} \quad \text { with } \rho \text { satisfying (0.3). }
\end{aligned}
$$

Under these conditions, our results are summarized in the following Theorem.

Theorem. Let $\left\{\phi^{-}, \psi^{-}\right\} \in \dot{H}^{1,2} \times L^{2}$ and

$$
w_{0}^{-}(t)=\cos \{H t\} \phi^{-}+H^{-1} \sin \{H t\} \psi^{-} .
$$

Then there exists $a \delta>0$ with the following properties.

(a) If $w_{0}{ }^{-}(t) \in V=V_{s, q}^{d}$ and $\left\|w_{0}{ }^{-}\right\|_{V} \leq \delta$, then there exists a unique solution $w(t) \in$ of $(0.5)$ which also satisfies

$$
\|w\|_{V} \leq \frac{4}{3}\left\|w_{0}{ }^{-}\right\|_{V} .
$$

(b) $w(t)$ satisfies the energy inequality 


$$
\|w(t)\|_{e^{2}}+\int_{\boldsymbol{R}^{n}} F(w(x, t)) d x \leq\left\|w_{0}^{-}(0)\right\|_{e^{2}} \text { for } t \in \boldsymbol{R},
$$

where $F(w)=\int_{0}^{w} f(u) d u$, and

$$
\left\|w(t)-w_{0}^{-}(t)\right\|_{e} \rightarrow 0 \text { as } t \rightarrow-\infty .
$$

(c) There exists $\left\{\phi^{+}, \phi^{+}\right\} \in \dot{H}^{1,2} \times L^{2}$ such that

$$
w_{0}{ }^{+}(t)=\cos \{H t\} \phi^{+}+H^{-1} \sin \{H t\} \phi^{+}
$$

belongs to $V$ and satisfies

$$
\left\|w_{0}{ }^{+}(t)\right\|_{e}=\left\|w_{0}{ }^{-}(t)\right\|_{e} \text { for } \quad t \in \boldsymbol{R}
$$

and

$$
\left\|w(t)-w_{0}^{+}(t)\right\|_{e} \rightarrow 0 \text { as } t \rightarrow+\infty .
$$

The correspondence $S:\left\{\phi^{-}, \phi^{-}\right\} \rightarrow\left\{\phi^{+}, \phi^{+}\right\}$defines the scattering operator.

Remark 1.1. If $\left\{\phi^{-}, \phi^{-}\right\} \in\left\{H^{1+s, q} \times H^{s, q}\right\} \cap\left\{H^{1, q / \rho} \times L^{q / \rho}\right\}$, we have $\left\{\phi^{-}\right.$, $\left.\psi^{-}\right\} \in \dot{H}^{1,2} \times L^{2}$ and $w_{0}^{-}(t) \in V$.

Remark 1.2. The conditions $\left\{\phi^{-}, \phi^{-}\right\} \in \dot{H}^{1,2} \times L^{2}$ and $w_{0}^{-}(t) \in V$ imply $\left\{\phi^{-}, \psi^{-}\right\} \in H^{s, q} \times H^{s-1, q}$. In fact, we have $\phi^{-}=w_{0}^{-}(0) \in H^{s, q}$ and $\phi^{-} \in L^{2} \subseteq$ $H^{s-1, q}$ (cf., Proposition 2.3 (i) of $\S 2$ ).

\section{§ 2. Existence and Uniqueness of Solutions}

We begin with well known results for the elementary solution $H^{-1} \sin$ $\{H t\}$ of the free wave equation (0.4).

Proposition 2.1 ( $L^{p}-L^{p^{\prime}}$ estimates). (i) Let $2 \leq q<\infty$ and $1 / q+1 / q^{\prime}$ $=1$. Then we have for $\phi \in C_{0}^{\infty}\left(\boldsymbol{R}^{n}\right)$,

$$
\left\|H^{-1} \exp \{i H t\} \phi\right\|_{q} \leq C|t|^{-d}\|\psi\|_{-s, q^{\prime}}^{\cdot},
$$

where $s$ and $d$ are given in (0.9).

(ii) Let $2 \leq q \leq 2(n+1) /(n-1)$. Then

$$
\left\|H^{-1} \exp \{i H t\} \psi\right\|_{q} \leq C|t|^{-b}\|\phi\|_{q^{\prime}} .
$$

where $-b=-n+1+2 n / q$.

See Pecher [8], Theorem 2.2 for a proof.

Proposition 2.2 ( $L^{p}-L^{p}$ estimates). Let $1<q<\infty$. If $|1 / q-1 / 2| \leq$ 
$1 /(n-1)$, we have

$$
\begin{gathered}
\left\|H^{-1} \sin \{H t\} \phi\right\|_{q} \leq C|t|\|\psi\|_{q}, \\
\|\cos \{H t\} \phi\|_{q} \leq C(1+|t|)\|\phi\|_{1, q} .
\end{gathered}
$$

See Peral [9], Theorems 1 and 2 for a proof.

Our main tools will be the following Sobolev embedding theorem:

Proposition 2.3. (i) Let $1<p \leq q<\infty$ and $s, t \in \mathbb{R}$. If $1 / p-s / n \leq 1 /$ $q-t / n$, then we have

$$
H^{s, p} \hookrightarrow H^{t, q} .
$$

(ii) If $1 / p-s / n=1 / q-t / n$, then we have

$$
\dot{H}^{s, p} \hookrightarrow \dot{H}^{t, q} \text {. }
$$

(iii) If $1<p<\infty$ and $s \geq 0$, then we have

$$
H^{s, p} \hookrightarrow L^{p} \cap \dot{H}^{s, p} \hookrightarrow H^{s, p} .
$$

Here $A \hookrightarrow B$ means that $A$ is continuously embedded in $B$.

Proof. (i) and (ii) are well known. See e.g., Tribel [11] § 2.8, BerghLöfström [1] Chapter 6 and Hörmander [4] Theorem 4.5.3 for a proof. On the other hand, (iii) is proved by use of the Mihlin multiplier theorem. For details, see Bergh-Löfström [1] Theorem 6.1.6.

Let $h(x) \in C_{0}{ }^{\infty}\left(\boldsymbol{R}^{n}\right)$ be a non-negative, even function satisfying $\int_{R^{n}} h(x) d x=1$. Put

$$
h_{j}(x)=\left\{\begin{array}{cc}
j^{n} h(j x) & j \in \mathbb{N} \\
\delta(x) & j=\infty,
\end{array}\right.
$$

where $\delta(x)$ is the Dirac function. For $u \in L^{p}(p \geq 1)$, we denote by $h_{j} * u$ the convolution of $h_{j}$ and $u$ :

$$
\left(h_{j} * u\right)(x)=\int_{R^{n}} h_{j}(x-y) u(y) d y .
$$

For each $-\infty \leq \sigma \leq \infty$ and $j \in \mathbb{N} \cup\{\infty\}$ we consider the integral equation

$$
w_{j}^{\sigma}(t)=h_{j} * w_{0}(t)-\int_{\sigma}^{t} H^{-1} \sin \{H(t-\tau)\} f_{j}\left(w_{j}^{\sigma}(\tau)\right) d \tau
$$

where 


$$
w_{0}(t)=\cos \{H t\} \phi+H^{-1} \sin \{H t\} \phi
$$

and

$$
f_{j}(w)=h_{j} * f\left(h_{j} * w\right)
$$

Note that (2.9) coincides with (0.5) if $w_{0}(t)=w_{0}{ }^{-}(t), \sigma=-\infty$ and $j=\infty$.

In this section we treat the solvability of (2.9) in the space $V=V_{s, q}^{d}$ with $q$ satisfying the following conditions :

$$
\begin{aligned}
& \frac{1}{q}-\frac{s}{n} \leq \frac{1}{\rho \tilde{q}^{\prime}} \leq \frac{1}{q}, \\
& \frac{1}{q}-\frac{s}{n} \leq \frac{1}{\rho q^{\prime}} \leq \frac{1}{q}, \\
& \frac{1}{\rho}<d<1,
\end{aligned}
$$

where $s=n+1 / q-n-1 / 2, d=(n-1)(1 / 2-1 / q), 1 / q^{\prime}=1-1 / q$ and

$$
\frac{1}{\tilde{q}^{\prime}}=\frac{1}{q^{\prime}}+\frac{s}{n} \text {. }
$$

Lemma 2.4. (i) Conditions (2.12) (2.14) are equivalent to

$$
q \leq \frac{2(n \rho-1)}{n+1},\left(1-\frac{n-1}{2 n} \rho\right) q \geq 1-\frac{\rho}{n} \text { and } q>\frac{2(n-1) \rho}{(n-1) \rho-2},
$$

and are satisfied by the pair $(q, \rho)$ given in (0.3) and (0.9).

(ii) (2.15), (2.12) and (2.13) imply the embeddings

$$
\begin{aligned}
& L^{\tilde{q}^{\prime} \hookrightarrow} \dot{H}^{-s, q^{\prime}}, \\
& H^{s, q} \hookrightarrow L^{\rho \tilde{q}^{\prime}} \cap L^{\rho q^{\prime}} .
\end{aligned}
$$

Proof. (i) As is easily seen, (2.12) (2.14) are reduced to the following three inequalities:

$$
\frac{1}{\rho \tilde{q}^{\prime}} \leq \frac{1}{q}, \quad \frac{1}{q}-\frac{s}{n} \leq \frac{1}{\rho q^{\prime}} \text { and } \frac{1}{\rho}<d,
$$

from which (2.16) follows. The lower bound $\gamma(n)$ of $(0.3)$ comes from the first and third inequalities of (2.16). On the other hand, the upper bound $(n+3) /(n-1)$ of $(0.3)$ is obtained by the first and second inequalities of (2.16). The rest of assertion (i) is obvious.

(ii) (2.17) and (2.18) follow from (2.5) and (2.6), respectively.

Remark 2.5. The second inequality of (2.13) and the first inequality of 
(2.14) yield the condition

$$
\rho>\rho(n)=\frac{n+1+\sqrt{n^{2}+10 n-7}}{2(n-1)} .
$$

The number $\rho(n)$ is known to be the critical power for global existence of solutions of (0.1) (see John [5] and Glassey [3]). Of course we have $\rho(n)<$ $\gamma(n)$.

Lemma 2.6. For any $p \geq 1$ and $u, v \in L^{\rho p}$, we have

$$
\left\|f_{j}(u)-f_{j}(v)\right\|_{p} \leq C\left(\|u\|_{\rho p}^{\rho-1}+\|v\|_{\rho p}^{\rho-1}\right)\|u-v\|_{\rho p},
$$

where $C>0$ is independent of $j \in N \cup\{\infty\}$. Moreover,

$$
\left\|f_{j}(u)-f(u)\right\|_{p} \rightarrow 0 \text { as } \quad j \rightarrow \infty .
$$

Proof. By (A2), the Young inequality and the Hölder inequality we have

$$
\begin{aligned}
\| f_{j}(u) & -f_{j}(v)\left\|_{p} \leq\right\| f\left(h_{j} * u\right)-f\left(h_{j} * v\right) \|_{p} \\
& \leq C\left(\left\|h_{j} * u\right\|_{\rho p}^{\rho-1}\left\|h_{j} * v\right\|_{\rho p}^{\rho-1}\right)\left\|h_{j} *(u-v)\right\|_{\rho p} \\
& \leq C\left(\|u\|_{\rho p}^{\rho-1}+\|v\|_{\rho p}^{\rho-1}\right)\|u-v\|_{\rho p}
\end{aligned}
$$

proving (2.20). Similarly, we have

$$
\left\|f_{j}(u)-f(u)\right\|_{p} \leq C\|u\|_{\rho p}^{\rho-1}\left\|h_{j} * u-u\right\|_{\rho p}+\left\|h_{j} * f(u)-f(u)\right\|_{p},
$$

from which (2.21) follows.

Combining (2.1) and (2.20), we can prove the

Lemma 2.7. Under conditions (2.12) and (2.13) we have

$$
\left\|H^{-1} \exp \{i H t\}\left\{f_{j}(u)-f_{j}(v)\right\}\right\|_{s, q} \leq C|t|^{-d}\left(\|u\|_{s, q}^{\rho-1}+\|v\|_{s, q}^{\rho-1}\right)\|u-v\|_{s, q},
$$

where $C>0$ is independent of $j \in N \cup\{\infty\}$.

Proof. Since $s \geq 0$, we can apply (2.1) and (2.17) to obtain

$$
\begin{aligned}
\left\|H^{-1} \exp \{i H t\} \psi\right\|_{s, q} & \leq C\left(\left\|H^{-1} \exp \{i H t\} \phi\right\|_{q}+\left\|H^{-1} \exp \{i H t\} \phi\right\|_{s, q}^{\cdot}\right) \\
& \leq C|t|^{-d}\left(\|\phi\|_{-s, q^{\prime}}^{\cdot}+\|\phi\|_{q^{\prime}}\right) \\
& \leq C|t|^{-d}\left(\|\psi\|_{\tilde{q}^{\prime}}+\|\phi\|_{q^{\prime}}\right) .
\end{aligned}
$$

We put $\psi=f_{j}(u)-f_{j}(v)$ in this inequality. It then follows from (2.20) and (2.18) that

$$
\|\phi\|_{\tilde{q}^{\prime}}+\|\psi\|_{q^{\prime}}=\left\|f_{j}(u)-f_{j}(v)\right\|_{\tilde{q}^{\prime}}+\left\|f_{j}(u)-f_{j}(v)\right\|_{q^{\prime}}
$$




$$
\begin{aligned}
& \leq C\left(\|u\|_{\rho \bar{q}^{\prime}}^{\rho-1}+\|v\|_{\rho}^{\rho} \tilde{q}^{-1}\right)\|u-v\|_{\rho \bar{q}^{\prime}}+C\left(\|u\|_{\rho} \rho_{\bar{q}^{\prime}}^{\rho-1}+\|v\|_{\rho q^{\prime}}^{\rho-1}\right)\|u-v\|_{\rho q^{\prime}} \\
& \leq C\left(\|u\|_{s, q}^{\rho-1}+\|v\|_{s, p}^{\rho-1}\right)\|u-v\|_{s, q} .
\end{aligned}
$$

Thus, we have (2.22).

We are now ready to prove the following

Proposition 2.8. Let $\{\phi, \phi\} \in H^{1,2} \times L^{2}$ and $w_{0}(t)$ be defined by (2.10). There exists $a \delta_{1}>0$ independent of $-\infty \leq \sigma \leq \infty$ and $j \in N \cup\{\infty\}$ with the following properties: Let $w_{0}(t) \in V$ and $\left\|w_{0}\right\|_{V} \leq 3 \delta_{1} / 4$, where $V$ is the Banach space (0.8) defined for $q$ satisfying (2.16). Then there exists a unique solution $w_{j}{ }^{\sigma}(t) \in V$ of (2.9), which also satisfies

$$
\left\|w_{j}^{\sigma}\right\|_{V} \leq \frac{4}{3}\left\|w_{0}\right\|_{V}
$$

Proof. For $u(t) \in V$ put

$$
\Phi_{j}{ }^{\sigma} u(t)=h_{j} * w_{0}(t)-\int_{\sigma}^{t} H^{-1} \sin \{H(t-\tau)\} f_{j}(u(\tau)) d \tau .
$$

We then have from (2.22)

$$
\begin{gathered}
\left\|\Phi_{j}{ }^{\sigma} u(t)-\Phi_{j}{ }^{\sigma} v(t)\right\|_{s, q} \\
\leq C \int_{\sigma}^{t}|t-\tau|^{-d}(1-|\tau|)^{-d \rho} d \tau\left(\|u\|_{V}^{\rho-1}+\|v\|_{V}^{\rho-1}\right)\|u-v\|_{V} .
\end{gathered}
$$

By (2.14)

$$
\int_{\sigma}^{t}|t-\tau|^{-d}(1+|\tau|)^{-d \rho} d \tau \leq \int_{-\infty}^{\infty}|t-\tau|^{-d}(1+|\tau|)^{-d \rho} d \tau \leq C(1+|t|)^{-d} .
$$

Hence it follows that

$$
\left\|\Phi_{j}{ }^{\sigma} u-\Phi_{j}{ }^{\sigma} v\right\|_{v} \leq \tilde{C}\left(\|u\|_{v}{ }^{\rho-1}+\|v\|_{v}{ }^{\rho-1}\right)\|u-v\|_{v} .
$$

Noting (A1) and $\left\|h_{j} * w_{0}\right\|_{s, q} \leq\left\|w_{0}\right\|_{s, q}$, we have similarly

$$
\left\|\Phi_{j}{ }^{\sigma} u\right\|_{v} \leq\left\|w_{0}\right\|_{v}+\tilde{C}\|u\|_{v}{ }^{\rho} .
$$

Now choose $\delta_{1}>0$ very small to satisfy $2 \tilde{C} \delta_{1}{ }^{\rho-1}=1 / 2$ and put $B\left(\delta_{1}\right)=\{u$ $\left.\in V ;\|u\|_{V} \leq \delta_{1}\right\}$. Then for $u, v \in B\left(\delta_{1}\right)$ we have from (2.26) and (2.27)

$$
\begin{gathered}
\left\|\Phi_{j}{ }^{\sigma} u-\Phi_{j}{ }^{\sigma} v\right\|_{V} \leq \frac{1}{2}\|u-v\|_{V}, \\
\left\|\Phi_{j}{ }^{\sigma} u\right\|_{V} \leq\left\|w_{0}\right\|_{V}+\frac{1}{4}\|u\|_{V} \leq \delta_{1}
\end{gathered}
$$


if $w_{0}$ is chosen to satisfy $\left\|w_{0}\right\|_{V} \leq 3 \delta_{1} / 4$. These show that each $\phi_{j}{ }^{\sigma}$ gives a contraction mapping on $B\left(\delta_{1}\right)$. Hence there exists a unique fixed point $w_{j}{ }^{\sigma}(t) \in B\left(\delta_{1}\right)$, which solves $(2.9)$.

(2.24) is obvious from (2.29).

\section{§ 3. Energy Inequalities}

In this section we use Proposition 2.2 and Proposition 2.1 (ii) to obtain energy inequalities corresponding to (0.12) of each solution $w_{j}{ }^{\sigma}(t) \in V$ of (2.9). To this end we require the condition $1 \leq \rho \leq(n+3) /(n-1)$. So, in the following the pair $(q, \rho)$ is restricted to satisfy $(0.3)$ and $(0.9)$.

Lemma 3.1. (i) For any $\ell \geq 0, j \in N$ and $\psi \in L^{p}(p \geq 1)$,

$$
\left\|H^{\ell} h_{j} * \psi\right\|_{q} \leq\left\|H^{\ell} h_{j}\right\|_{r}\|\psi\|_{p}
$$

if $r \geq 1$ is chosen to satisfy $1 / q=1 / r+1 / p-1$.

(ii) For any $\ell \geq 0, j \in \mathbb{N}$ and $u, v \in H^{s, q}$,

$$
\left\|H^{\ell} f_{j}(u)-H^{\ell} f_{j}(v)\right\|_{p} \leq C\left\|H^{\ell} h_{j}\right\|_{r}\left(\|u\|_{s, q}^{\rho-1}+\|v\|_{s, q}^{\rho-1}\right)\|u-v\|_{s, q}
$$

if $p \geq 1$ and $r \geq 1$ are chosen to satisfy $1 / q=1 / r+1 / q^{\prime}-1$.

Proof. (i) is the so called Young inequality.

(ii) By (i) and (2.20) we have

$$
\begin{aligned}
& \left\|H^{\ell} f_{j}(u)-H^{\ell} f_{j}(v)\right\|_{p} \leq C\left\|H^{\ell} h_{j}\right\|_{r}\left\|f\left(h_{j} * u\right)-f\left(h_{j} * v\right)\right\|_{q^{\prime}} \\
\leq & C\left\|H^{\ell} h_{j}\right\|_{r}\left(\left\|h_{j} * u\right\|_{\rho q^{\prime}}^{\rho-1}+\left\|h_{j} * v\right\|_{\rho q^{\prime}}^{\rho-1}\left\|h_{j} * u-h_{j} * v\right\|_{\rho q^{\prime}}\right. \\
\leq & C\left\|H^{\ell} h_{j}\right\|_{r}\left(\|u\|_{\rho q^{\prime}}^{\rho-1}+\|v\|_{\rho q^{\prime}}^{\rho-1}\right)\|u-v\|_{\rho q^{\prime}} .
\end{aligned}
$$

Thus, the embedding (2.18) yilds (3.2).

Lemma 3.2. Let $\{\phi, \phi\} \in\left(\dot{H}^{1,2} \cap L^{q}\right) \times L^{2}$. Then we have for any $j$ $\in N$,

$$
h_{j} * w_{0}(t)=\cos \{H t\} h_{j} * \phi+H^{-1} \sin \{H t\} h_{j} * \phi
$$

and

$$
\partial_{t}\left\{h_{j} * w_{0}(t)\right\}=-H \sin \{H t\} h_{j} * \phi+\cos \{H t\} h_{j} * \psi \text { in } L^{q} .
$$

Proof. (3.3) is obvious if we note $\{\phi, \phi\} \in L^{q} \times L^{2}$ in (2.10). To show (3.4), let us first put 


$$
J_{1}(\eta)=\left[\frac{\cos \{H(t+\eta)\}-\cos \{H t\}}{\eta}+H \sin \{H t\}\right] h_{j} * \phi .
$$

Then by the mean value theorem

$$
J_{1}(\eta)=\int_{0}^{1} \int_{0}^{1} \theta \eta H^{2} \cos \left\{H\left(t+\theta \theta^{\prime} \eta\right)\right\} h_{j} * \phi d \theta^{\prime} d \theta .
$$

Thus, from (2.4) of Proposition 2.2 and (3.1) with $p=q$ it follows that

$$
\begin{aligned}
\left\|J_{1}(\eta)\right\|_{q} \leq & |\eta| \int_{0}^{1} \int_{0}^{1}\left\|\cos \left\{H\left(t+\theta \theta^{\prime} \eta\right)\right\} H^{2} h_{j} * \phi\right\|_{q} d \theta^{\prime} d \theta \\
\leq & |\eta| \int_{0}^{1} \int_{0}^{1} C\left(1+\left|t+\theta \theta^{\prime} \eta\right|\right) d \theta^{\prime} d \theta \\
& \times\left(\left\|H^{2} h_{j}\right\|_{1}+\left\|H^{3} h_{j}\right\|_{1}\right)\|\phi\|_{q} \rightarrow 0 \text { as } \eta \rightarrow 0 .
\end{aligned}
$$

Next put

$$
J_{2}(\eta)=\left[\frac{H^{-1} \sin \{H(t+\eta)\}-H^{-1} \sin \{H t\}}{\eta}-\cos \{H t\}\right] h_{j} * \psi .
$$

Then from (2.3) of Proposition 2.2 and (3.1) with $p=2$ it follows that

$$
\left\|J_{2}(\eta)\right\|_{q} \leq|\eta| \int_{0}^{1} \int_{0}^{1} C\left|t+\theta \theta^{\prime} \eta\right| d \theta^{\prime} d \theta\left\|H^{2} h_{j}\right\|_{r}\|\psi\|_{2} \rightarrow 0 \text { as } \eta \rightarrow 0 .
$$

Hence, we see that (3.4) holds true.

Lemma 3.3. Let $\sigma \in \boldsymbol{R}$ and $j \in \boldsymbol{N}$. Then for $w_{j}^{\sigma}(t) \in V$ of Proposition 2.8 we have

$$
\begin{gathered}
w_{j}{ }^{\sigma}(t) \in C_{t}{ }^{1}\left(\boldsymbol{R}: L^{q}\right), \\
\partial_{t} w_{j}{ }^{\sigma}(t), H w_{j}{ }^{\sigma}(t) \in C_{t}{ }^{1}\left(\boldsymbol{R}: L^{2}\right) \cap C_{t}\left(\boldsymbol{R}: H^{1,2}\right) .
\end{gathered}
$$

Further, we have the differential equation

$$
\partial_{t}{ }^{2} w_{j}^{\sigma}(t)-\Delta w_{j}^{\sigma}(t)+f_{i}\left(w_{j}^{\sigma}(t)\right)=0 \text { in } L^{2} .
$$

Proof. We put

$$
\begin{aligned}
J_{3}(\eta) & =\frac{1}{\eta}\left[\int_{\sigma}^{t+\eta} H^{-1} \sin \{H(t+\eta-\tau)\} f_{j}\left(w_{j}^{\sigma}(\tau)\right) d \tau\right. \\
& \left.-\int_{\sigma}^{t} H^{-1} \sin \{H(t-\tau)\} f_{j}\left(w_{j}^{\sigma}(\tau)\right) d \tau\right]-\int_{\sigma}^{t} \cos \{H(t-\tau)\} f_{j}\left(w_{j}^{\sigma}(\tau)\right) d \tau .
\end{aligned}
$$

Applying (3.2) with $p=q, u=w_{j}^{\sigma}$ and $v=0$, we have

$$
\left\|J_{3}(\eta)\right\|_{q} \leq \frac{1}{|\eta|}\left|\int_{t}^{t+\eta} C\right| t+\eta-\tau\left|\left\|f_{j}\left(w_{j}^{\sigma}(\tau)\right)\right\|_{q} d \tau\right|
$$




$$
\begin{aligned}
& +|\eta| \int_{0}^{1} \int_{0}^{1}\left|\int_{\sigma}^{t} C\right| t-\tau+\theta \theta^{\prime} \eta\left|\left\|H^{2} f_{j}\left(w_{j}^{\sigma}(\tau)\right)\right\|_{q} d \tau\right| d \theta^{\prime} d \theta \\
& \leq C|\eta|\left\{\left\|h_{j}\right\|_{q / 2}+(|t-\sigma|+|\eta|)^{2}\left\|H^{2} h_{j}\right\|_{q / 2}\right\}\left\|w_{j}^{\sigma}\right\|_{v}{ }^{\rho} \\
& \rightarrow 0 \text { as } \eta \rightarrow 0 .
\end{aligned}
$$

Since $w_{j}^{\sigma}(t)$ satisfies (2.9), this and (3.4) show

$$
\begin{aligned}
\partial_{t} w_{j}^{\sigma}(t)= & -H \sin \{H t\} h_{j} * \phi+\cos \{H t\} h_{j} * \phi \\
& -\int_{\sigma}^{t} \cos \{H(t-\tau)\} f_{i}\left(w_{j}^{\sigma}(\tau)\right) d \tau \text { in } L^{q} .
\end{aligned}
$$

from which (3.5) follows.

Next choose $p=2$ and $1 / r=1 / q+1 / 2$ in (3.2). Then

$$
\begin{aligned}
& \left\|f_{j}\left(w_{j}^{\sigma}(t)\right)-f_{j}\left(w_{j}^{\sigma}\left(t^{\prime}\right)\right)\right\|_{\ell, 2} \\
& \quad \leq C\left\|h_{j}\right\|_{\ell, r}\left\|f\left(h_{j} * w_{j}^{\sigma}(t)\right)-f\left(h_{j} * w_{j}^{\sigma}\left(t^{\prime}\right)\right)\right\|_{q^{\prime}} \\
& \quad \leq C\left\|h_{j}\right\|_{\ell, r}\left(\left\|w_{j}^{\sigma}(t)\right\|_{s, p}^{\rho-1}+\left\|w_{j}^{\sigma}\left(t^{\prime}\right)\right\|_{s, q}^{\rho-1}\right)\left\|w_{j}^{\sigma}(t)-w_{j}^{\sigma}\left(t^{\prime}\right)\right\|_{s, q},
\end{aligned}
$$

and it follows that

$$
f_{j}\left(w_{j}^{\sigma}(t)\right) \in C_{t}\left(\boldsymbol{R}: H^{\ell, 2}\right) \text { for any } \ell \geq 0 .
$$

Applying this and Lemma 3.2 to (2.9), we obtain (3.6).

Finally, (3.7) follows from (3.6) and (3.9).

Lemma 3.4. (i) We have the embedding

$$
H^{s, q} \hookrightarrow L^{\rho+1}
$$

(ii) For each $-\infty \leq \sigma \leq \infty$ and $j \in \mathbb{N} \cup\{\infty\}$ we have

$$
\left|\int_{R^{n}} F\left(h_{j} * w_{j}^{\sigma}(x, t)\right) d x\right| \leq C(1+|t|)^{-d(\rho+1)}\left\|w_{0}\right\|_{V}^{\rho+1} .
$$

(iii) Let $\sigma \in \mathbb{R}$ and $j \in \mathbb{N}$. Then

$$
\frac{d}{d t} \int_{R^{n}} F\left(h_{j} * w_{j}^{\sigma}(x, t)\right) d x=\left(\partial_{t} w_{j}^{\sigma}(t), f_{j}\left(w_{j}^{\sigma}(t)\right)\right)_{2},
$$

where $(,)_{2}$ denotes the inner product of $L^{2}$.

Note that the double convolution mollifier (2.11) is usefull in obtaining relation (3.12) (see Ginibre-Velo [2]).

Proof. (i) The first inequality of (2.12) and the second inequality of (2.13) are reduced to the condition 


$$
\frac{1}{q}-\frac{s}{n} \leq \frac{1}{\rho+1} \leq \frac{1}{q} .
$$

This gives the above embedding.

(ii) (3.11) directly follows from (3.10) and (2.24) if we note the Young inequality $\left\|h_{j} * w_{j}^{\sigma}(t)\right\|_{\rho+1} \leq\left\|h_{j}\right\|_{1}\left\|w_{j}^{\sigma}(t)\right\|_{\rho+1}$.

(iii) Let us consider

$$
\begin{aligned}
J_{4}(\eta)= & \frac{1}{\eta} \int_{R^{n}}\left\{F\left(h_{j} * w_{j}^{\sigma}(t+\eta)\right)-F\left(h_{j} * w_{j}^{\sigma}(t)\right)\right\} d x \\
& -\left(\partial_{t} w_{j}^{\sigma}(t), f_{j}\left(w_{j}^{\sigma}(t)\right)\right)_{2} .
\end{aligned}
$$

By the mean value theorem

$$
\begin{aligned}
& F\left(h_{j} * w_{j}{ }^{\sigma}(t+\eta)\right)-F\left(h_{j} * w_{j}^{\sigma}(t)\right) \\
& =\int_{0}^{1} f\left(\theta h_{j} * w_{j}^{\sigma}(t+\eta)+(1-\theta) h_{j} * w_{j}^{\sigma}(t)\right) d \theta \\
& \quad \times h_{j} *\left\{w_{j}^{\sigma}(t+\eta)-w_{j}^{\sigma}(t)\right\} .
\end{aligned}
$$

$h$ being even, this implies that

$$
\begin{aligned}
J_{4}(\eta)= & \frac{1}{\eta} \int_{0}^{1} d \theta \int_{R^{n}}\left\{f_{j}\left(\theta w_{j}{ }^{\sigma}(t+\eta)+(1-\theta) w_{j}{ }^{\sigma}(t)\right)\left\{w_{j}{ }^{\sigma}(t+\eta)-w_{j}{ }^{\sigma}(t)\right\} d x\right. \\
& -\int_{R^{n}} \partial_{t} w_{j}{ }^{\sigma}(t) f_{j}\left(w_{j}{ }^{\sigma}(t)\right) d x .
\end{aligned}
$$

Applying the Hölder inequality, we then have

$$
\begin{aligned}
\left|J_{4}(\eta)\right| \leq & \int_{0}^{1} d \theta\left\{\left\|f_{j}\left(\theta w_{j}{ }^{\sigma}(t+\eta)+(1-\theta) w_{j}^{\sigma}(t)\right)\right\|_{q^{\prime}}\right. \\
& \times\left\|\frac{1}{\eta}\left\{w_{j}^{\sigma}(t+\eta)-w_{j}^{\sigma}(t)\right\}-\partial_{t} w_{j}^{\sigma}(t)\right\|_{q} \\
& \left.+\left\|f_{j}\left(\theta w_{j}{ }^{\sigma}(t+\eta)+(1-\theta) w_{j}{ }^{\sigma}(t)\right)-f_{j}\left(w_{j}{ }^{\sigma}(t)\right)\right\|_{q^{\prime}}\left\|\partial_{t} w_{j}{ }^{\sigma}(t)\right\|_{q}\right\} .
\end{aligned}
$$

Hence, noting (3.5) and (3.2) with $p=q^{\prime}$, we can let $\eta \rightarrow 0$ to obtain (3.12). $\square$

We can now prove the

Proposition 3.5. Let $\sigma \in \boldsymbol{R}$ and $j \in N$. The following relation holds for any $t \in \boldsymbol{R}$.

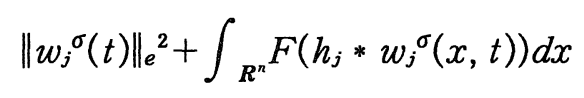




$$
=\left\|h_{j} * w_{0}(\sigma)\right\|_{e}^{2}+\int_{R^{n}} F\left(h_{j} * h_{j} * w_{0}(x, \sigma)\right) d x .
$$

Proof. By Lemmas 3.3 and 3.4 (iii) we can show

$$
\frac{d}{d t}\left\{\left\|w_{j}^{\sigma}(t)\right\|_{e^{2}}+\int_{R^{n}} F\left(h_{j} * w_{j}^{\sigma}(x, t)\right) d x\right\}=0
$$

which proves (3.14) since we have $w_{j}^{\sigma}(\sigma)=h_{j} * w_{0}(\sigma)$.

Next we let $j \rightarrow \infty$ in (3.14).

Lemma 3.6. For $j=\infty$ and $-\infty \leq \sigma \leq \infty$ we denote $w^{\sigma}(t)=w_{\infty}{ }^{\sigma}(t)$. Then $w^{\sigma}(t) \in C_{t}{ }^{1}\left(\mathbb{R}: \mathcal{S}^{\prime}\right)$ and

$$
\begin{gathered}
H w^{\sigma}(t)=\cos \{H t\} H \phi+\sin \{H t\} \phi-\int_{\sigma}^{t} \sin \{H(t-\tau)\} f\left(w^{\sigma}(\tau)\right) d \tau \\
\partial_{t} w^{\sigma}(t)=-\sin \{H t\} H \phi+\cos \{H t\} \psi-\int_{\sigma}^{t} \cos \{H(t-\tau)\} f\left(w^{\sigma}(\tau)\right) d \tau .
\end{gathered}
$$

Proof. (3.15) is obvious. To show (3.16) we consider the functional $\left(w_{0}(t), \zeta\right)_{2}$ in $\zeta \in \mathcal{S}$. Since $H \phi, \phi \in L^{2}$, it follows that

$$
\begin{aligned}
\frac{d}{d t}\left(w_{0}(t), \zeta\right)_{2} & =(\phi,-H \sin \{H t\} \zeta)_{2}+(\phi, \cos \{H t\} \zeta)_{2} \\
& =(-\sin \{H t\} H \phi+\cos \{H t\} \psi, \zeta)_{2} .
\end{aligned}
$$

Next put

$$
\begin{aligned}
K(\eta)= & \frac{1}{\eta}\left[\int_{\sigma}^{t+\eta}\left(H^{-1} \sin \{H(t+\eta-\tau)\} f\left(w^{\sigma}(\tau)\right), \zeta\right)_{2} d \tau\right. \\
& \left.-\int_{\sigma}^{t}\left(H^{-1} \sin \{H(t-\tau)\} f\left(w^{\sigma}(\tau)\right), \zeta\right)_{2} d \tau\right] \\
& -\int_{\sigma}^{t}\left(\cos \{H(t-\tau)\} f\left(w^{\sigma}(\tau)\right), \zeta\right)_{2} d \tau
\end{aligned}
$$

Then by an argument similar to Lemma 3.3, we have

$$
\begin{aligned}
\mid K(\eta) \leq & \frac{1}{|\eta|}\left|\int_{t}^{t+\eta}\left\|f\left(w^{\sigma}(\tau)\right)\right\|_{q^{\prime}} C\right| t+\eta-\tau\left|\|\zeta\|_{q} d \tau\right| \\
& +|\eta| \int_{0}^{1} \int_{0}^{1}\left|\int_{\sigma^{t}}^{t}\left\|f\left(w^{\sigma}(\tau)\right)\right\|_{q^{\prime}} C\right| t-\tau+\left.\theta \theta^{\prime} \eta\right|^{-d}\left\|H^{2} \zeta\right\|_{\tilde{q}^{\prime}} d \tau \mid d \theta d \theta^{\prime}
\end{aligned}
$$

where $\tilde{q}^{\prime}(=q / \rho)$ is given by (2.15). Since

$$
\left\|f\left(w^{\sigma}(\tau)\right)\right\|_{q^{\prime}} \leq C(1+|\tau|)^{-d \rho}\left\|w^{\sigma}\right\|_{V^{\prime}}
$$


this shows that

$$
\begin{aligned}
& \frac{d}{d t}\left(\int_{\sigma}^{t} H^{-1} \sin \{H(t-\tau)\} f\left(w^{\sigma}(\tau)\right) d \tau, \zeta\right)_{2} \\
& =\left(\int_{\sigma}^{t} \cos \{H(t-\tau)\} f\left(w^{\sigma}(\tau)\right) d \tau, \zeta\right)_{2} .
\end{aligned}
$$

Summarizing these results, we obtain (3.16).

Lemma 3.7. Let $\sigma \in \boldsymbol{R}$. We have for each $t \in \boldsymbol{R}$,

$$
\left\|w_{j}^{\sigma}(t)-w^{\sigma}(t)\right\|_{\rho+1} \rightarrow 0 \text { as } j \rightarrow \infty .
$$

Proof. By (2.9)

$$
\begin{aligned}
w_{j}^{\sigma}(t)-w^{\sigma}(t) & =h_{j} * w_{t}(t)-w_{t}(t) \\
& -\int_{\sigma}^{t} H^{-1} \sin \{H(t-\tau)\}\left\{f_{j}\left(w_{j}^{\sigma}(\tau)\right)-f_{j}\left(w^{\sigma}(\tau)\right)\right\} d \tau \\
& -\int_{\sigma}^{t} H^{-1} \sin \{H(t-\tau)\}\left\{f_{j}\left(w^{\sigma}(\tau)\right)-f\left(w^{\sigma}(\tau)\right)\right\} d \tau \\
& =I_{j 1}(t)+I_{j 2}(t)+I_{j 3}(t) .
\end{aligned}
$$

Since $w_{0}(t) \in H^{s, q} \subseteq L^{\rho+1}$ by (3.10), it is obvious that

$$
\left\|I_{j 1}(t)\right\|_{\rho+1} \rightarrow 0 \text { as } j \rightarrow \infty \text {. }
$$

To estimate $I_{j 3}(t)$ we use (2.2) of Proposition 2.1 with $q=\rho+1$. Then

$$
\left\|I_{j 3}(t)\right\|_{\rho+1} \leq\left|\int_{\sigma}^{t} C\right| t-\left.\tau\right|^{-b}\left\|f_{j}\left(w^{\sigma}(\tau)\right)-f\left(w^{\sigma}(\tau)\right)\right\|_{(\rho+1) / \rho} d \tau \mid
$$

and (2.21) with $p=(\rho+1) / \rho$ and the Lebesque dominated convergence theorem show

$$
\left\|I_{j 3}(t)\right\|_{\rho+1} \rightarrow 0 \text { as } j \rightarrow \infty \text {. }
$$

For $I_{j 2}(t)$ we have from (2.2) and (2.20)

$$
\begin{gathered}
\left\|I_{j 2}(t)\right\|_{\rho+1} \leq C\left|\int_{\sigma}^{t}\right| t-\left.\tau\right|^{-b}\left\{\left\|w_{j}^{\sigma}(\tau)\right\|_{\rho+1}^{\rho-1}+\left\|w^{\sigma}(\tau)\right\|_{\rho+1}^{\rho-1}\right\} \\
\times\left\|w_{j}^{\sigma}(\tau)-w^{\sigma}(\tau)\right\|_{\rho+1} d \tau \mid \\
\leq C\left\|w_{0}\right\|_{V}^{\rho-1}\left|\int_{\sigma}^{t}\right| t-\left.\tau\right|^{-b}(1+|\tau|)^{-d(\rho-1)}\left\|w_{j}^{\sigma}(\tau)-w^{\sigma}(\tau)\right\|_{\rho+1} d \tau \mid .
\end{gathered}
$$

Here in the last inequality we have used (3.10) and (2.24).

Summarizing the argument, we see the following: For any $\varepsilon>0$ there 
exists a $j_{0}>0$ such that

$$
\begin{aligned}
& \left\|w_{j}^{\sigma}(t)-w^{\sigma}(t)\right\|_{\rho+1} \\
& \quad \leq C\left\|w_{0}\right\|_{V}^{\rho-1}\left|\int_{\sigma}^{t}\right| t-\left.\tau\right|^{-b}\left\|w_{j}^{\sigma}(\tau)-w^{\sigma}(\tau)\right\|_{\rho+1} d \tau \mid+\varepsilon
\end{aligned}
$$

for any $j \geq j_{0}$. Hence, by the Gronwall inequality we have

$$
\left\|w_{j}^{\sigma}(t)-w^{\sigma}(t)\right\|_{\rho+1} \leq \varepsilon \exp \left\{C\left\|w_{0}\right\|_{v}^{\rho-1}\left|\int_{\sigma}^{t}\right| t-\left.\tau\right|^{-b} d \tau \mid\right\} .
$$

(3.17) is thus proved.

Proposition 3.8. Let $\sigma \in \mathbb{R}$. The total energy of $w^{\sigma}(t)$ is estimated as follows :

$$
\begin{aligned}
& \left\|w^{\sigma}(t)\right\|_{e^{2}}+\int_{R^{n}} F\left(w^{\sigma}(x, t)\right) d x \\
& \quad \leq\left\|w_{0}(\sigma)\right\|_{e^{2}}+\int_{R^{n}} F\left(w_{0}(x, \sigma)\right) d x \quad \text { for } \quad t \in \mathbb{R} .
\end{aligned}
$$

Proof. The Hölder inequality, (3.10) and (2.24) show that

$$
\begin{aligned}
\mid \int_{R^{n}}\{ & \left.F\left(h_{j} * w_{j}^{\sigma}(t)\right)-F\left(w^{\sigma}(t)\right)\right\} d x \mid \leq C(1+|t|)^{-d \rho}\left\|w_{0}\right\|_{V}^{\rho} \\
& \times\left\{\left\|h_{j} *\left\{w_{j}^{\sigma}(t)-w^{\sigma}(t)\right\}\right\|_{\rho+1}+\left\|h_{j} * w^{\sigma}(t)-w^{\sigma}(t)\right\|_{\rho+1}\right\} \rightarrow 0
\end{aligned}
$$

as $j \rightarrow \infty$ and

$$
\begin{aligned}
& \left|\int_{R^{n}}\left\{F\left(h_{j} * h_{j} * w_{0}(\sigma)\right)-F\left(w_{0}(\sigma)\right)\right\} d x\right| \\
& \leq C\left\|w_{0}(\sigma)\right\|_{s, q}^{\rho}\left\|h_{j} * h_{j} * w_{0}(\sigma)-w_{0}(\sigma)\right\|_{\rho+1} \rightarrow 0
\end{aligned}
$$

as $j \rightarrow \infty$. Thus, we can let $j \rightarrow \infty$ in (3.14) to obtain

$$
\begin{aligned}
& \lim _{j \rightarrow \infty}\left\|w_{j}^{\sigma}(t)\right\|_{e^{2}}+\int_{R^{n}} F\left(w^{\sigma}(x, t)\right) d x \\
& =\left\|w_{0}(\sigma)\right\|_{e^{2}}+\int_{\mathbb{R}^{n}} F\left(w_{0}(x, \sigma)\right) d x .
\end{aligned}
$$

On the other hand, we have from Lemmas 3.6 and 3.7

$$
H w_{j}^{\sigma}(t) \rightarrow H w^{\sigma}(t) \text { in } \mathcal{S}^{\prime} \text {. }
$$

Further, we have from Lemmas 2.6 and 2.7

$$
\partial_{t} w_{j}^{\sigma}(t) \rightarrow \partial_{t} w^{\sigma}(t) \text { in } \mathcal{S}^{\prime} .
$$

In fact 
$\left\|\partial_{t} h_{j} * w_{0}(t)-\partial_{t} w_{0}(t)\right\|_{2} \leq\left\|h_{j} * H \phi-H \phi\right\|_{2}+\left\|h_{j} * \psi-\psi\right\|_{2}$ and for any $\zeta \in S$.

$$
\begin{aligned}
& \left|\left(\partial_{t} \int_{\sigma}^{t} H^{-1} \sin \{H(t-\tau)\}\left\{f_{i}\left(w_{j}^{\sigma}(\tau)\right)-f\left(w^{\sigma}(\tau)\right)\right\} d \tau, \zeta\right)_{2}\right| \\
& \quad \leq\left|\int_{\sigma}^{t}\left\|f_{j}\left(w_{j}^{\sigma}(\tau)\right)-f\left(w^{\sigma}(\tau)\right)\right\|_{(\rho+1) / \rho}\|\cos \{H(t-\tau)\} \zeta\|_{\rho+1} d \tau\right| \\
& \quad \leq\|\xi\|_{(\rho+1) / \rho}\left|\int_{\sigma}^{t}\left\|f_{j}\left(w_{j}^{\sigma}(\tau)\right)-f\left(w^{\sigma}(\tau)\right)\right\|_{(\rho+1) / \rho} d \tau\right| .
\end{aligned}
$$

(3.19) (3.21) show that as $j \rightarrow \infty$

$$
w_{j}^{\sigma}(t) \rightarrow w^{\sigma}(t) \text { weakly in the energy norm. }
$$

Combining (3.19) and (3.22), we obtain (3.18).

Finally, we let $\sigma \rightarrow \pm \infty$ in (3.18).

Lemma 3.9. We have for any $t \in \boldsymbol{R}$,

$$
\left\|w^{\sigma}(t)-w^{ \pm \infty}(t)\right\|_{s, q} \rightarrow 0 \text { as } \sigma \rightarrow \pm \infty \text {. }
$$

Proof. Using the argument of Proposition 3.6 of [7], we have

$$
\begin{aligned}
\left\|w^{\sigma}(t)-w^{ \pm \infty}(t)\right\|_{s, q} & \leq \tilde{C}(1+|t|)^{-d}\left\{\left\|w^{\sigma}\right\|_{V}^{\rho-1}+\left\|w^{ \pm \infty}\right\|_{V}^{\rho-1}\right\}\left\|w^{\sigma}-w^{ \pm \infty}\right\|_{V} \\
& +C_{\varepsilon}(1+|\sigma|)^{-\varepsilon}(1+|t|)^{-d}\left\|w^{ \pm \infty}\right\|_{V}{ }^{\rho}
\end{aligned}
$$

for $\varepsilon$ satisfying $0<\varepsilon<d \rho-1$ ( $\widetilde{C}$ being the constant given in the proof of Proposition 2.8). Since $\widetilde{C}\left\{\left\|w^{\sigma}\right\|_{v}^{\rho-1}+\left\|w^{ \pm \infty}\right\|_{v}^{\rho-1}\right\}<1 / 2$, this implies (3.21).

Proposition 3.10. The total energy of $w^{ \pm \infty}(t)$ is estimated as follows :

$$
\left\|w^{ \pm \infty}(t)\right\|_{e^{2}}+\int_{\boldsymbol{R}^{n}} F\left(w^{ \pm \infty}(x, t)\right) d x \leq\left\|w_{0}(0)\right\|_{e^{2}} \text { for } \quad t \in \boldsymbol{R} .
$$

Proof. As in the proof of Proposition 3.8, we have

$$
\left|\int_{R^{n}}\left\{F\left(w^{\sigma}(t)\right)-F\left(w^{ \pm \infty}(t)\right)\right\} d x\right| \leq C(1+|t|)^{-d \rho}\left\|w_{0}\right\|_{V}{ }^{\rho}\left\|w^{\sigma}(t)-w^{ \pm \infty}(t)\right\|_{s, q}
$$

and

$$
\left|\int_{\boldsymbol{R}^{n}} F\left(w_{0}(\sigma)\right) d x\right| \leq C\left\|w_{0}(\sigma)\right\|_{s, q}^{\rho+1} \leq C(1+|\sigma|)^{-d(\rho+1)}\left\|w_{0}\right\|_{V^{\rho+1}}
$$

Thus, letting $\sigma \rightarrow \pm \infty$ in (3.18), we obtain

$$
\limsup _{\sigma \rightarrow \pm \infty}\left\|w^{\sigma}(t)\right\|_{e^{2}}+\int_{R^{n}} F\left(w^{ \pm \infty}(t)\right) d x \leq\left\|w_{0}(0)\right\|_{e^{2}} .
$$


With this inequality, the rest of proof of $(3.24)_{ \pm}$is same to the above Proposition 3.8 .

\section{$\S 4$. Proof of Theorem}

Let $U_{0}(t), t \in \mathbb{R}$, be the unitary group in the energy space $\dot{H}^{1,2} \times L^{2}$ defined by

$$
\begin{aligned}
U_{0}(t)\left\{u_{1}, u_{2}\right\}=\left\{\cos \{H t\} u_{1}\right. & +H^{-1} \sin \{H t\} u_{2}, \\
& \left.-H \sin \{H t\} u_{1}+\cos \{H t\} u_{2}\right\} .
\end{aligned}
$$

This $U_{0}(t)$ gives the solution of the free equation (0.4). Namely for $w_{0}(t)$ of $(2.10)$ we have

$$
\left\{w_{0}(t), \partial_{t} w_{0}(t)\right\}=U_{0}(t)\{\phi, \phi\} .
$$

Lemma 4. 1 . (i) $U_{0}(t)(t \neq 0)$ is continuously extended to the map $H^{1, q^{\prime}} \times L^{q^{\prime}} \rightarrow H^{s, q} \times H^{s-1, q}$ and we have

$$
\left\|U_{0}(t)\left\{u_{1}, u_{2}\right\}\right\|_{H^{s . q \times} \times H^{s-1 . q}} \leq C\left(|t|^{-d}+|t|^{-b}\right)\left\|\left\{u_{1}, u_{2}\right\}\right\|_{H^{1, q^{\prime} \times L^{q^{q}}} .} .
$$

(ii) $U_{0}(t), t \in \mathbb{R}$, is continuously extended to the map $H^{s, q} \times H^{s-1, q} \rightarrow$ $H^{s-1, q} \times H^{s-2, q}$ and we have for $\left\{u_{1}, u_{2}\right\} \in H^{1, q^{\prime}} \times L^{q^{\prime}}$,

$$
U_{0}(t) U_{0}\left(t^{\prime}\right)\left\{u_{1}, u_{2}\right\}=U_{0}\left(t+t^{\prime}\right)\left\{u_{1}, u_{2}\right\} \quad\left(t^{\prime} \neq 0, t+t^{\prime} \neq 0\right) .
$$

Proof. (i) (4.3) easily follows from (2.1) and (2.2).

(ii) By Proposition 2.2 we have similarly

$$
\left\|U_{0}(t)\left\{u_{1}, u_{2}\right\}\right\|_{H^{s-1, q} X_{H} s_{-2, q}} \leq C(1+|t|)\left\{\left\|u_{1}\right\|_{s, q}+\left\|u_{2}\right\|_{s-1-q}\right\},
$$

which implies the first assertion. (4.4) is proved as follows: For any $\left\{u_{1}\right.$, $\left.u_{2}\right\} \in H^{1, q^{\prime}} \times L^{q^{\prime}},\left\{h_{j} * u_{1}, h_{j} * u_{2}\right\} \in\left\{H^{1, q^{\prime}} \times L^{q^{\prime}}\right\} \cap\left\{\dot{H}^{1,2} \times L^{2}\right\}$ and converges as $j \rightarrow \infty$ to $\left\{u_{1}, u_{2}\right\}$ in $H^{1, q^{\prime}} \times L^{q^{\prime}}$. Then by (i) and the first assertion of (ii)

$$
\begin{aligned}
& U_{0}(t) U_{0}\left(t^{\prime}\right)\left\{u_{1}-h_{j} * u_{1}, u_{2}-h_{j} * u_{2}\right\} \rightarrow 0 \text { in } H^{s-1, q} \times H^{s-2, q}, \\
& U_{0}\left(t+t^{\prime}\right)\left\{h_{j} * u_{1}, h_{j} * u_{2}\right\} \rightarrow U_{0}\left(t+t^{\prime}\right)\left\{u_{1}, u_{2}\right\} \text { in } H^{s, q} \times H^{s-1, q},
\end{aligned}
$$

and we have (4.4).

Now we note $\{\phi, \phi\} \in H^{s, q} \times H^{s-1, q}$ and $\left\{0, f\left(w^{ \pm \infty}(t)\right)\right\} \in H^{1, q^{\prime}} \times L^{q^{\prime}}$ (see Remark 1.2 and Lemma 2.4). Then by means of the above lemma, we have from (2.9) and (3.16)

$$
\left\{w^{ \pm \infty}(t), \partial_{t} w^{ \pm \infty}(t)\right\}=U_{0}(t)\{\phi, \phi\}-\int_{ \pm \infty}^{t} U_{0}(t-\tau)\left\{0, f\left(w^{ \pm \infty}(\tau)\right)\right\} d \tau
$$


$(t \gtrless 0)$ in $H^{s-1, q} \times H^{s-2, q}$, and further

$$
U_{0}(-t)\left\{w^{ \pm \infty}(t), \partial_{t} w^{ \pm \infty}(t)\right\}=\{\phi, \phi\}-\int_{ \pm \infty}^{t} U_{0}(-\tau)\left\{0, f\left(w^{ \pm \infty}(\tau)\right)\right\} d \tau
$$

$(t \gtrless 0)$ in $S^{\prime} \times S^{\prime}$ since we have $U_{0}(-t) U_{0}(t)\{\phi, \phi\}=\{\phi, \phi\}$ in $\dot{H}^{1,2} \times L^{2}$.

Proposition 4. 2. We have

$$
\left\|w^{ \pm \infty}(t)-w_{0}(t)\right\|_{e} \rightarrow 0 \text { as } t \rightarrow \pm \infty .
$$

Proof. By (4.6) and (2.22)

$$
\begin{aligned}
\left\|U_{0}(-t)\left\{w^{ \pm \infty}(t), \partial_{t} w^{ \pm \infty}(t)\right\}-\{\phi, \psi\}\right\|_{H^{s, q \times H} H^{s-1, q}} \\
\quad \leq\left. C\left|\int_{ \pm \infty}^{t}\right| \tau\right|^{-d \rho} d \tau \mid\left\|w^{ \pm \infty}\right\|_{\nu}{ }^{\rho} \rightarrow 0 \text { as } t \rightarrow \pm \infty,
\end{aligned}
$$

Note that

$$
\begin{aligned}
& \left\|U_{0}(-t)\left\{w^{ \pm \infty}(t), \partial_{t} w^{ \pm \infty}(t),\right\}\right\|_{H^{1,2} \times L^{2}}^{2} \\
& \quad=\left\|H w^{ \pm \infty}(t)\right\|_{2}{ }^{2}+\left\|\partial_{t} w^{ \pm \infty}(t)\right\|_{2}{ }^{2}=2\left\|w^{ \pm \infty}(t)\right\|_{e^{2}}
\end{aligned}
$$

and

$$
\int_{R^{n}} F\left(w^{ \pm \infty}(x, t)\right) d x \rightarrow 0 \text { as } t \rightarrow \pm \infty
$$

by (3.11). Then the energy estimates $(3.24)_{ \pm}$and (4.8) imply

$$
U_{0}(-t)\left\{w^{ \pm \infty}(t), \partial_{t} w^{ \pm \infty}(t)\right\} \rightarrow\{\phi, \phi\} \text { weakly in } \dot{H}^{1,2} \times L^{2}
$$

as $t \rightarrow \pm \infty$, and we have using again (3.24) \pm and (4.9)

$$
\begin{aligned}
\left\|w_{0}(0)\right\|_{e^{2}} & \leq \frac{1}{2} \liminf _{t \rightarrow \pm \infty}\left\|\left\{w^{ \pm \infty}(t), \partial_{t} w^{ \pm \infty}(t)\right\}\right\|_{H^{1,2} \times L^{2}}^{2} \\
& =\liminf _{t \rightarrow \pm \infty}\left\{\left\|w^{ \pm \infty}(t)\right\|_{e^{2}}+\int_{R^{n}} F\left(w^{ \pm \infty}(t)\right) d x\right\} \leq\left\|w_{0}(0)\right\|_{e^{2}}^{2} .
\end{aligned}
$$

Combining this and (4.10), we conclude the strong convergence (4.7) $)_{ \pm} \square$

Proof of Theorem. (a) We put

$$
\delta=\frac{1}{2} \delta_{1},
$$

where $\delta_{1}$ is given in Proposition 2.8. Since $\left\{\phi^{-}, \psi^{-}\right\} \in \dot{H}^{1,2} \times L^{2}$ and $\left\|w_{0}^{-}\right\|_{V}$ $\leq \delta \leq 3 \delta_{1} / 4$, (0.5), i.e., (2.9) with $w_{0}(t)=w_{0}^{-}(t), \sigma=-\infty$ and $j=\infty$, has a unique solution $w(t)=w^{-\infty}(t) \in V$. (1.2) is also proved in Proposition 2.8.

(b) The energy estimate (1.3) is proved in (3.24) - of Proposition 3.10, 
and (1.4) is known in (4.7)- of Proposition 4.2.

(c) We put

$$
\left\{\phi^{+}, \psi^{+}\right\}=\left\{\phi^{-}, \psi^{-}\right\}-\int_{-\infty}^{\infty} U_{0}(-\tau)\{0, f(w(\tau))\} d \tau,
$$

where $w(t)=w^{-\infty}(t)$ given above. We then have from (4.6)

$$
\left\{\phi^{+}, \phi^{+}\right\}=U_{0}(-t)\left\{w(t), \partial_{t} w(t)\right\}-\int_{t}^{\infty} U_{0}(-\tau)\{0, f(w(\tau))\} d \tau .
$$

By the argument of the proof of Proposition 4.2 we see that $\left\{\phi^{+}, \phi^{+}\right\} \in \dot{H}^{1,2}$ $\times L^{2}$. Moreover, we have

$$
w_{0}{ }^{+}(t)=w_{0}{ }^{-}(t)-\int_{-\infty}^{\infty} H^{-1} \sin \{H(t-\tau)\} f(w(\tau)) d \tau \in V,
$$

and hence by $(2.27)$

$$
\left\|w_{0}{ }^{+}\right\|_{V} \leq\left\|w_{0}{ }^{-}\right\|_{V}+C\|w\|_{V} \leq \frac{1}{2} \delta_{1}+\frac{1}{4} \delta_{1}=\frac{3}{4} \delta_{1},
$$

where $w_{0}{ }^{+}(t)$ is given by (1.5). Thus, $w(t)$ in (4.13) coincides with the solution $w^{+\infty}(t) \in V$ of $(2.9)$ with $w_{0}(t)=w_{0}{ }^{+}(t), \sigma=+\infty$ and $j=\infty$ constructed in Proposition 2.8. In this sence, (1.7) is already known in (4.7)+.

Finally, if we let $t \rightarrow-\infty$ in $(3.24)_{+}$with $w_{0}(t)=w_{0}{ }^{+}(t)$, then it follows from (1.4) and (4.9) that

$$
\left\|w_{0}{ }^{-}(0)\right\|_{e^{2}} \leq\left\|w_{0}{ }^{+}(0)\right\|_{e^{2}} .
$$

On the other hand, if we let $t \rightarrow+\infty$ in (1.3), then it follows from (4.7)+ and (4.9) that

$$
\left\|w_{0}{ }^{+}(0)\right\|_{e^{2}} \leq\left\|w_{0}{ }^{-}(0)\right\|_{e^{2}} .
$$

These prove (1.6), and the proof of Theorem is completed.

\section{References}

[1] Bergh, J. and Löfström, J., Interpolation Spaces, Berlin-Heidelberg-New York, Springer 1976.

[ 2 ] Ginibre, J. and Velo, G., On a class of nonlinear Schrödinger equation I, J. Funct. Analysis, 32 (1979), 1-32.

[ 3 ] Glassey, R. T., Finite-time blow-up for solutions of nonlinear wave equation, Math. Z., 177 (1981), 323-340.

[4] Hörmander, L., The Analysis of Linear Partial Differential Operators I, BerlinHeidelberg-New York, Springer 1983.

[5] John, F., Blow-up of solutions of nonlinear wave equations in three dimensions, Manu- 
scripta Math., 28 (1979), 235-268.

[6] Klainerman, S., Long time behavior of the solutions to nonlinear evolution equations, Arch. Rat. Mech. Analysis, 78 (1982), 73-98.

[ 7 ] Mochizuki, K. and Motai, T., The scattering theory for the nonlinear wave equation with small data, J. Math. Kyoto Univ., 25 (1985), 703-715.

[ 8] Pecher, H., $L^{p}$-Abschätzungen und Klassische Lösungen für Nichtlineare Wellengleichungen I, Math. Z., 150 (1976), 159-183.

[ 9] Peral, J. C., $L^{p}$ estimates for the wave equation, J. Funct. Analysis, 36 (1980), 114-145.

[10] Strauss, W. A., Nonlinear scattering theory at low energy, J. Funct. Analysis, 41 (1981), 110-133.

[11] Triebel, H., Interpolation Theory, Function Spaces, Differential Operators, AmsterdamNew York-Oxford, North-Holland 1978.

Added in proof. Recently, pecher [12] showed that a densely defined scattering operator exists for equation (0.1) with $f(w)=\lambda(w)^{p-1} w$ if $\mathrm{n}=3$ and $3 \geq p \geq p(3)=1+\sqrt{2}$. He used a weighted norm in space-time originally introduced in [5] and it seems not so easy to extend his results to other dimensions, especially to $n \geq 4$.

[12] Pecher, H., Scattering for semilinear wave equations with small data in three dimensions, Preprint 1987. 